\title{
Evaluation of sample preservation and storage methods for metaproteomics analysis of intestinal microbiomes
}

\author{
Angie Mordant ${ }^{1}$, Manuel Kleiner ${ }^{1}$
}

Affiliations:

1. Department of Plant and Microbial Biology, North Carolina State University, Raleigh, NC, USA

\section{Correspondence:}

Angie Mordant - almordan@ncsu.edu

Manuel Kleiner - mkleine@ncsu.edu

\section{Abstract}

A critical step in studies of the intestinal microbiome using meta-omics approaches is the preservation of samples before analysis. Preservation is essential for approaches that measure gene expression, such as metaproteomics, which is used to identify and quantify proteins in microbiomes. Intestinal microbiome samples are typically stored by flash freezing and storage at $-80^{\circ} \mathrm{C}$, but some experimental set-ups do not allow for immediate freezing of samples. In this study, we evaluated methods to preserve fecal microbiome samples for metaproteomics analyses when flash freezing is not possible. We collected fecal samples from C57BL/6 mice and stored them for 1 and 4 weeks using the following methods:

flash-freezing in liquid nitrogen, immersion in RNAlater ${ }^{\mathrm{TM}}$, immersion in 95\% ethanol, immersion in a RNAlater-like buffer, and combinations of these methods. After storage we extracted protein and prepared peptides for LC-MS/MS analysis to identify and quantify peptides and proteins. All samples produced highly similar metaproteomes, except for ethanol-preserved samples that were distinct from all other samples in terms of protein identifications and protein abundance profiles. Flash-freezing and RNAlater ${ }^{\mathrm{TM}}$ 
(or RNAlater-like treatments) produced metaproteomes that differed only slightly, with less than $0.7 \%$ of identified proteins differing in abundance. In contrast, ethanol preservation resulted in an average of $9.5 \%$ of the identified proteins differing in abundance between ethanol and the other treatments. Our results suggest that preservation at room temperature in RNAlater ${ }^{\mathrm{TM}}$, or an RNAlater-like solution, performs as well as freezing for the preservation of intestinal microbiome samples before metaproteomics analyses.

\section{Importance}

Metaproteomics is a powerful tool to study the intestinal microbiome. By identifying and quantifying a large number of microbial, dietary, and host proteins in microbiome samples, metaproteomics provides direct evidence of the activities and functions of microbial community members. A critical step for metaproteomic workflows is preserving samples before analysis because protein profiles are susceptible to fast change in response to changes in environmental conditions (air exposure, temperature changes, etc.). This study evaluated the effects of different preservation treatments on the metaproteomes of intestinal microbiome samples.

\section{Introduction}

The intestinal microbiome is a highly diverse and metabolically active community that has profound effects on its host [1]. This complex community influences the health of its host by altering the availability of nutrients [2-5] and the host's susceptibility to infection and disease [6, 7]. The intestinal microbiome is integral to proper host immune function [8-10] and host well-being [11, 12]. So far most studies have used DNA sequencing and taxonomy-based approaches to study the intestinal microbiome providing critical insights into taxonomic shifts in the community-related host genotype, diet, and disease state [13-16]. Taxonomic shifts, however, have been found to not always measure important functional shifts in the microbiome, as different taxa can perform the same function [17] and highly similar strains can perform different functions by encoding a few unique gene clusters [18]. Therefore the use of 
function-focused multi-omics approaches is essential for understanding the role of the intestinal microbiome in health and disease [19-22].

Metaproteomics is a valuable tool to study interactions in the intestinal microbiome and the microbiome's influence on host health [23-25]. Metaproteomics allows for the identification and quantification of large numbers of microbial, dietary, and host proteins in microbiome samples in a high-throughput fashion [26, 26-28]. Because proteins are central to all biological processes, metaproteomics provides direct evidence of the activities and functions of microbial community members and their contributions to disease [29]. For example, metaproteomics revealed protein biomarkers of disease in Inflammatory Bowel Disease (IBD) and colorectal cancer and provided insights into the role of the microbiome in such diseases [30, 31]. In addition to quantifying differences in protein abundances between samples, metaproteomics can also be used to assess microbial community structure based on proteinaceous biomass [32-35] and track incorporation of specific substrates using stable isotope content of peptides [36-39].

Metaproteomics workflows are complex and variability can be introduced at every step, from sample preparation to data acquisition by LC-MS/MS and data processing [35]. There is no standardized workflow for metaproteomics of intestinal microbiome samples but some of the individual steps have been optimized in the past, such as protein extraction [40], database creation [41], and database searching [42]. A critical step that has not yet been optimized is the storage and preservation of samples before analysis. Adequate storage of samples is critical because exposure to environmental changes could induce changes in protein profiles of species in the samples and thus provide misleading study results. For example, exposing samples to air can strongly bias colorectal cancer studies because oxidative stress and enrichment of bacterial superoxide dismutase enzymes that will occur from air exposure are also characteristics of colorectal cancer in the intestinal tract [30]. Therefore, appropriately storing samples immediately upon collection helps to avoid post-collection changes in protein abundances. A suitable storage method should preserve the information contained in the microbiome at the time of sampling without introducing substantial bias. 
Typically, microbiome samples are frozen immediately upon collection, with flash-freezing in liquid nitrogen followed by storage at $-80^{\circ} \mathrm{C}$. However, flash-freezing is not always possible, and very little is known about suitable alternatives to flash-freezing for the preservation of microbiome samples before metaproteomics analyses. For example, clinical or diet studies involving human subjects usually require the subjects to perform the sampling themselves at their homes where they do not have access to liquid nitrogen $[43,44]$. It can be difficult to maintain sample integrity also in resource-limited fieldwork conditions [45] or out in a wild animal's environment where there is no access to liquid nitrogen and cold storage [46]. Even in the laboratory, immediate freezing in liquid nitrogen can be difficult. One specific case is work with gnotobiotic animals, which are invaluable models to study and manipulate the microbiota in a controlled system. Gnotobiotic animals reside in isolators where everything (food, bedding, etc) entering the isolators is sterilized through autoclaving, irradiation, or strong chemicals before being introduced through a two-ended port [47]. Removing samples from the isolators causes long delays between sampling and sample storage, thus exposing samples to environmental changes (air exposure, temperature change, nutrient depletion, etc) before they can be adequately stored.

Several studies have been conducted to test the effects of preservation methods on nucleic acids, but the effects of such methods on proteins are poorly understood. Freezing at $-80^{\circ} \mathrm{C}$ is thought to maintain sample integrity similar to fresh samples $[48,49]$. The effectiveness of storage at room temperature is typically evaluated based on comparisons to frozen treatments. RNAlater ${ }^{\mathrm{TM}}$ is a popular storage solution that has been shown to be effective at preserving DNA in gut microbiome studies, with negligible differences compared to freezing $[50,51]$. Ethanol (95\% or absolute) may also be suitable for the preservation of nucleic acids in microbiome samples before taxonomic profiling as long as it is used consistently $[45,46,52]$. However, there are studies in which these storage solutions significantly biased the downstream results [53], particularly in RNA sequencing studies [54] and thus great care should be taken when selecting a preservation method. While the effects of sample preservation on nucleic acids have been extensively studied, to the best of our knowledge, only two studies have investigated the effects of sample preservation on protein profiles. First, Saito and colleagues demonstrated that RNAlater ${ }^{\mathrm{TM}}$ has the potential to preserve proteomes as effectively as immediate freezing [55]. Their 
results are promising, however, their study was performed on a single marine microorganism (cyanobacterium Synechococcus WH8102) and thus does not indicate whether RNAlater ${ }^{\mathrm{TM}}$ would preserve samples as complex as those from the intestinal microbiome. Furthermore, their study was performed in earlier stages of proteomics when replication was expensive and effort-intensive. For that reason, the researchers only included technical replication and therefore they could not assess the robustness of RNAlater ${ }^{\mathrm{TM}}$ in terms of within-treatment consistency. Second, Hickl and colleagues observed vast differences in the identifications and relative abundances of proteins from human fecal samples depending on the preservation and storage procedure applied to the samples [56]. They tested two preservation and storage methods: a flash-freezing-based approach "FF" and RNAlater, "RL". The first method "FF", consisted of flash-freezing in liquid nitrogen followed by storage at $-80^{\circ} \mathrm{C}$, cryomilling, and storage at $-20^{\circ} \mathrm{C}$ for $16 \mathrm{~h}$ while immersed in RNAlaterICE $®$. The "RL" method simply consisted of immersion in refrigerated RNAlater ${ }^{\mathrm{TM}}$ for $6 \mathrm{~h}$. They found less than $50 \%$ overlap in protein identifications between the two treatments. Of the overlapping proteins, they found roughly 2,000 proteins to significantly differ in abundance by more than 1.5 fold between the two treatments. The majority of the differences they observed were attributed to taxonomy. For example, class Actinobacteria represented about $20 \%$ of the composition of the FF samples while Actinobacteria only made up less than $2 \%$ of the composition of the RL samples. However, one cannot attribute the differences Hickl et al. observed to a specific aspect of the preservation and storage process because of the many variables in the sample processing. For example, the flash-frozen samples were cryomilled which could favor lysis of gram-positive bacteria, such as Actinobacteria, and may explain the large difference in the relative abundance of this taxon [57].

This study aimed to (1) compare the effects of different sample preservation methods on intestinal microbiome metaproteomes, (2) evaluate comparable aspects of sample processing by limiting the number of variables, (3) assess within-treatment variability, and (4) evaluate the methods over a longer period of preservation/storage time.

\section{Materials and Methods}

\section{Preparation of fecal master mix and preservation treatments}


Fresh fecal pellets were collected from 12 conventional 5-month old C57BL/6 mice obtained from the Jackson Laboratory. To remove inter-individual variation as a variable, the pellets were pooled and homogenized using a spatula to make a fecal master mix. The master mix was split into aliquots of $8 \mathrm{mg}$ each. The aliquots were either resuspended in $200 \mu \mathrm{L}$ of a preservation solution and stored at room temperature $\left(22^{\circ} \mathrm{C}\right)$ in the dark, or were flash-frozen using liquid nitrogen and stored at $-80^{\circ} \mathrm{C}$. The preservation treatments are discussed in detail below. In brief, we tested 6 preservation treatments: flash-freezing in liquid nitrogen followed by storage at $-80^{\circ} \mathrm{C}$ ("FF" treatment); preservation at room temperature in RNAlater ${ }^{\mathrm{TM}}$ (RNAlater ${ }^{\mathrm{TM}}$ Stabilization Solution, Invitrogen; "R" treatment); a combination of RNAlater ${ }^{\mathrm{TM}}$ and flash freezing ("RF" treatment); an RNAlater ${ }^{\mathrm{TM}}$-like preservation buffer ("NAP buffer") as described in [58] ("N" treatment); this same "NAP buffer" autoclaved ("AN" treatment); and 95\% ethanol ("E" treatment). We tested the effectiveness of the treatments at preserving microbiome samples over two storage durations: 1 week and 4 weeks. We prepared four replicate samples per treatment and time point, with each replicate being an $8 \mathrm{mg}$ aliquot of the fecal master mix described above.

\section{Flash-freezing and storage at $-80^{\circ} \mathrm{C}$ ("FF")}

Immediate freezing followed by storage at $-80^{\circ} \mathrm{C}$ is the method most frequently used to preserve biological specimens and is regarded as the "gold-standard" approach. Fouhy and colleagues observed that immediate freezing retains information similar to fresh samples in a 16S rRNA gene amplicon sequencing experiment of healthy human fecal samples [48]. The only significant differences they observed between frozen and fresh samples were in the relative abundances of the genera Faecalibacterium and Leuconostoc; however, the differences were subtle and may be attributable to a batch effect in DNA extraction rather than sample preservation. The effectiveness of storage solutions used at room temperature is typically evaluated based on comparisons to frozen treatments [55].

\section{Immersion in RNAlater ${ }^{\mathrm{TM}}$ and storage at room temperature (“R”)}

RNAlater ${ }^{\mathrm{TM}}$ Stabilization Solution is a popular storage reagent. Its effectiveness can be attributed to its ability to quickly permeate tissue to stabilize and protect RNA. RNAlater ${ }^{\mathrm{TM}}$ is effective at preserving nucleic acids for intestinal microbiome studies, with negligible bias compared to freezing [50]. RNAlater ${ }^{\mathrm{TM}}$ 
has the potential to preserve proteins because its main component is ammonium sulfate, and ammonium sulfate precipitates proteins which can later be re-solubilized without degradation. Saito et al. demonstrated that RNAlater ${ }^{\mathrm{TM}}$ is effective at preserving the proteome of the marine cyanobacterium Synechococcus WH8102 [55]. In our study, we immersed samples in RNAlater ${ }^{\mathrm{TM}}$ (Invitrogen) in a 1:10 sample: solution ratio and then stored them at room temperature $\left(\sim 22^{\circ} \mathrm{C}\right)$ in the dark.

\section{Immersion in RNAlater ${ }^{\mathrm{TM}}$ and flash-freezing followed by storage at room temperature ("RF”)}

To determine whether the use of a storage solution makes a difference as compared to storing samples dry at $-80^{\circ} \mathrm{C}$, we immersed RF samples in RNAlater ${ }^{\mathrm{TM}}$ (Invitrogen) and flash-froze the tubes in liquid nitrogen before storing them at $-80^{\circ} \mathrm{C}$. Observed differences between $\mathrm{R}$ and RF samples would provide evidence regarding the effects of freezing on sample integrity.

\section{Immersion in NAP buffer and storage at room temperature ("N")}

The major limitation of RNAlater ${ }^{\mathrm{TM}}$ is its high cost. It has been demonstrated that RNAlater ${ }^{\mathrm{TM}}$-like buffers work as effectively as the commercially available solution [59]. Menke and colleagues even argue that their RNAlater-like solution called "Nucleic Acid Preservation (NAP) buffer" outperformed commercial RNAlater ${ }^{\mathrm{TM}}$ in preserving DNA for 16S rRNA gene amplicon sequencing experiments based on comparisons with immediately-frozen controls [58]. "NAP buffer" was included as a treatment in this study and was prepared as previously described by Camacho-Sanchez et al. [59]. Briefly, 1.5 L of NAP buffer (pH 5.2) contained $935 \mathrm{ml}$ of ultrapure water, $700 \mathrm{~g}$ of ammonium sulfate, $25 \mathrm{ml}$ of $1 \mathrm{M}$ sodium citrate, and $40 \mathrm{ml}$ of $0.5 \mathrm{M}$ ethylenediaminetetraacetic acid (EDTA). We prepared the solution fresh two days before the experiment. We immersed the samples in the NAP buffer solution in a 1:10 sample: solution ratio before storing them at room temperature $\left(\sim 22^{\circ} \mathrm{C}\right)$ in the dark.

\section{Immersion in Autoclaved NAP buffer and storage at room temperature ("AN")}

RNAlater ${ }^{\mathrm{TM}}$ and RNAlater-like buffers do not need to be autoclaved because their chemical composition inhibits the growth of contaminants. Manufacturers of RNAlater ${ }^{\mathrm{TM}}$ recommend against autoclaving the reagent. However, in some cases, such as when working with gnotobiotic isolators, the solution needs to 
be autoclaved to prevent the introduction of microorganisms into the isolators. We tested an autoclaved version of the NAP buffer as an additional treatment to simulate real experimental conditions with gnotobiotic isolators. The same solution described above as the $\mathrm{N}$ treatment, from the same batch, was autoclaved $\left(60 \mathrm{~min}\right.$ at $\left.121.5^{\circ} \mathrm{C}\right)$ two days before the start of the experiment. We immersed the samples in the autoclaved NAP buffer solution in a 1:10 sample: solution ratio before storing them at room temperature $\left(\sim 22^{\circ} \mathrm{C}\right)$ in the dark.

\section{Immersion in 95\% Ethanol and storage at room temperature ("E')}

Alcohol preservation is a common method in which biological specimens are preserved by dehydration. Hale and colleagues [46] found that absolute ethanol worked as well as immediate freezing of DNA for preserving samples prior to metagenomics analysis. The effectiveness of ethanol as a preservation treatment depends on its concentration. Sinha et al. [60] observed low stability of microbial DNA when preserved in $70 \%$ ethanol. Saito et al. [55] observed that $90 \%$ was not ideal for the preservation of the proteome of the marine cyanobacterium Synechococcus WH8102 as only $\sim 75 \%$ of the proteins were recovered as compared to flash-freezing. Because the organism studied by Saito et al. [55] is very different from the intestinal microbiome, we included ethanol (95\%) as a treatment. We prepared $95 \%$ ethanol by mixing pure anhydrous (200 proof/100\%) ethyl alcohol (Koptec) with ultrapure water (Optima ${ }^{\text {TM }}$ LC/MS Grade, Fisher Chemical ${ }^{\mathrm{TM}}$ ).

\section{Protein extraction and peptide preparation}

We prepared samples for metaproteomics analysis at two time-points: after storing the samples for 1 week and after 4 weeks. We removed the storage solutions from the samples by centrifugation at 21,000 $\mathrm{x} \mathrm{g}$ for $5 \mathrm{~min}$ and then resuspended the samples in $400 \mu \mathrm{l}$ of SDT lysis buffer [4\% (w/v) SDS, $100 \mathrm{mM}$ Tris-HCl pH 7.6, 0.1 M DTT]. Cells were lysed by bead-beating in lysing matrix E tubes (MP Biomedicals) with a Bead Ruptor Elite (Omni International) for 5 cycles of $45 \mathrm{sec}$ at $6.45 \mathrm{~m} / \mathrm{s}$ with $1 \mathrm{~min}$ dwell time between cycles, followed by heating at $95^{\circ} \mathrm{C}$ for $10 \mathrm{~min}$. The lysates were centrifuged for $5 \mathrm{~min}$ at 21,000 $\mathrm{xg}$ to remove cell debris. We prepared peptides according to the filter-aided sample preparation (FASP) protocol described by [61]. All centrifugations mentioned below were performed at $14,000 \times \mathrm{g}$. Samples 
were loaded onto $10 \mathrm{kDa}$ MWCO $500 \mu \mathrm{l}$ centrifugal filters (VWR International) by combining $60 \mu \mathrm{l}$ of lysate with $400 \mu$ l of Urea solution (8 M urea in $0.1 \mathrm{M} \mathrm{Tris/HCl} \mathrm{pH} \mathrm{8.5)} \mathrm{and} \mathrm{centrifuging} \mathrm{for} 30 \mathrm{~min}$. This step was repeated twice until the filter capacity was reached. Filters were washed twice by applying 200 $\mu \mathrm{l}$ of urea solution followed by $40 \mathrm{~min}$ of centrifugation. $100 \mu \mathrm{l}$ IAA solution (0.05 M iodoacetamide in Urea solution) was then added to filters for a $20 \mathrm{~min}$ incubation followed by centrifugation for $20 \mathrm{~min}$. The filters were washed three times with $100 \mu$ of urea solution and 20 min centrifugations, followed by buffer exchange to $\mathrm{ABC}$ (50 mM Ammonium Bicarbonate). Buffer exchange was accomplished by adding $100 \mu \mathrm{l}$ of $A B C$ and centrifuging three times followed by centrifugation for 20 min. Tryptic digestion was performed by adding $0.85 \mu \mathrm{g}$ of MS grade trypsin (Thermo Scientific Pierce, Rockford, IL, USA) in $40 \mu \mathrm{l}$ of ABC to the filters which and incubating for 16 hours in a wet chamber at $37^{\circ} \mathrm{C}$. The tryptic peptides were eluted by adding $50 \mu \mathrm{l}$ of $0.5 \mathrm{M} \mathrm{NaCl}$ and centrifuging for $20 \mathrm{~min}$. Peptide concentrations were determined with the Pierce Micro BCA assay (Thermo Fisher Scientific) following the manufacturer's instructions.

\section{LC-MS/MS}

Samples were analyzed by 1D-LC-MS/MS using a published method [62] with several modifications. The samples were blocked and randomized according to Oberg and Vitek's method [63] to control for batch effects. For each sample, 600 ng of tryptic peptides were loaded with an UltiMate ${ }^{\mathrm{TM}} 3000 \mathrm{RSLCnano}$ Liquid Chromatograph (Thermo Fisher Scientific) in loading solvent A (2 \% acetonitrile, $0.05 \%$ trifluoroacetic acid) onto a 5 mm, $300 \mu \mathrm{m}$ ID C18 Acclaim® PepMap100 pre-column and desalted (Thermo Fisher Scientific). Peptides were then separated on a $75 \mathrm{~cm}$ x $75 \mu \mathrm{m}$ analytical EASY-Spray column packed with PepMap RSLC C18, $2 \mu \mathrm{m}$ material (Thermo Fisher Scientific) heated to $60^{\circ} \mathrm{C}$ via the

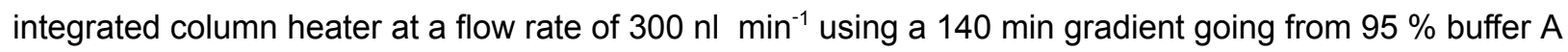
( $0.1 \%$ formic acid) to $31 \%$ buffer B (0.1\% formic acid, $80 \%$ acetonitrile $)$ in $102 \mathrm{~min}$, then to $50 \%$ B in 18 min, to $99 \%$ B in 1 min and ending with $99 \%$ B. Carryover was reduced by wash runs (injection of $20 \mu l$ acetonitrile with $99 \%$ eluent buffer B) between samples.

The analytical column was connected to a Q Exactive HF hybrid quadrupole-Orbitrap mass spectrometer (Thermo Fisher Scientific) via an Easy-Spray source. Eluting peptides were ionized via electrospray 
ionization (ESI). $\mathrm{MS}^{1}$ spectra were acquired by performing a full MS scan at a resolution of 60,000 on a 380 to $1600 \mathrm{~m} / \mathrm{z}$ window. $\mathrm{MS}^{2}$ spectra were acquired using a data-dependent approach by selecting for fragmentation the 15 most abundant ions from the precursor $\mathrm{MS}^{1}$ spectra. A normalized collision energy of 25 was applied in the HCD cell to generate the peptide fragments for $\mathrm{MS}^{2}$ spectra. Other settings of the data-dependent acquisition included: a maximum injection time of $100 \mathrm{~ms}$, a dynamic exclusion of $25 \mathrm{sec}$, and exclusion of ions of +1 charge state from fragmentation. About $60,000 \mathrm{MS} / \mathrm{MS}$ spectra were acquired per sample.

\section{Protein Identification Database}

We constructed a protein sequence database for identifying proteins from the four main components of the sample: the host, wheat (the main component of mouse chow), the microbiota, and potential contaminants. Protein sequences of the mouse host, Mus musculus, were downloaded from Uniprot (https://www.uniprot.org/proteomes/UP000000589). Protein sequences of wheat, Triticum aestivum, were downloaded from Uniprot (https://www.uniprot.org/proteomes/UP000019116 ). For the microbiota sequences, a public database constructed by Xiao et al. [64] was used. While the use of such a reference database is not recommended for studies that address specific biological questions, as it has been shown that such reference databases can lead to lower identification rates and species and protein miss assignments $[41,65]$, it is sufficient for determining the overall effects of sample preservation and preparation methods. The database from Xiao et al. contains $\sim 2.6$ million "non-redundant" genes from metagenomic sequencing of fecal material from 184 mice. The corresponding annotated protein sequences were downloaded from http://gigadb.org/dataset/view/id/100114/token/mZIMYJIF04LshpgP. The taxonomy (available as a separate file) was integrated into the string of the sequence descriptions using the join command in Linux. Most $(67.8 \%)$ of the sequences were assigned a taxonomy at the phylum level, and $9.8 \%$ of the sequences were assigned at the genus level [64]. Initial analyses suggested the presence of sequences that were too similar for adequate discrimination in the downstream workflow so the protein sequences were clustered with an identity threshold of $95 \%$ using the CD-HIT tool [66]. About $8 \%$ of the sequences were combined into clusters, while the remaining $\sim 92 \%$ remained as individual sequences. Also included in the database were sequences of common laboratory 
contaminants (http://www.thegpm.org/crap/). The database contained a total of 2,396,591 protein sequences and is included with the PRIDE submission for this study (PXD024115).

\section{Protein identification and quantification}

For peptide and protein identification, MS data were searched against the above-described database using the Sequest HT node in Proteome Discoverer version 2.3.0.523 (Thermo Fisher Scientific) with the following parameters: digestion with trypsin (Full), maximum of 2 missed cleavages, 10 ppm precursor mass tolerance, $0.1 \mathrm{Da}$ fragment mass tolerance and maximum 3 equal dynamic modifications per peptide. We considered the following dynamic modifications: oxidation on M (+15.995 Da), carbamidomethyl on $\mathrm{C}(+57.021 \mathrm{Da})$, and acetyl on the protein $\mathrm{N}$ terminus $(+42.011 \mathrm{Da})$. Peptide false discovery rate (FDR) was calculated using the Percolator node in Proteome Discoverer and only peptides identified at a 5\% FDR were retained for protein identification. Proteins were inferred from peptide identifications using the Protein-FDR Validator node in Proteome Discoverer with a target FDR of 5\%. From the generated multiconsensus dataset, we removed contaminant (cRAP sequences) and low confidence proteins (> $5 \%$ FDR) and kept proteins that were identified by at least 1 protein unique peptide. To decrease the number of "one-hit wonder" proteins, we removed proteins that were not detected in a total of at least 3 samples ( $n=47$ samples in total), which is the minimum number of replicates in one treatment and time-point. The dataset contained 6,086 proteins after applying these filtering steps. The data were then normalized by calculating normalized spectral abundance factors (NSAFs, [67]) and multiplied by 100 , to give the relative protein abundance in $\%$.

\section{Quality assessment and outlier analysis}

We assessed data quality by first inspecting raw data in the Xcalibur ${ }^{\mathrm{TM}}$ Software (Thermo Fisher Scientific), then by comparing the number of peptide spectrum matches (PSMs), peptides, proteins, and protein groups identified in each sample individually. We tested for statistical significance using a Student's t-test (2-tailed, equal variability, FDR of 0.05). Samples in the dataset had on average 25,220 +/- 4.954 proteins identified at 5\% FDR and 3,499 +/- 662 identified protein groups. Assuming the numbers of proteins identified per sample were normally distributed data, about $99.7 \%$ of the samples in 
the dataset were expected to have at least 10,358 detected proteins and 1,513 protein groups. These numbers correspond to the means stated above minus three standard deviations. One ethanol-preserved sample ("E6") was deemed an outlier and was removed from the dataset because it had only 767 proteins and 84 protein groups. We suspect the protein extraction for that particular sample failed due to leaks in the filter unit during sample preparation.

\section{Data analyses}

To investigate the degree of overlap in protein identifications between treatments, we used the filtered dataset of 6,086 proteins. If a protein was detected in at least one sample of a treatment within this dataset, it was counted as identified in that treatment. We imported the accession codes of the identified proteins into Venny 2.1 [68] to create Venn diagrams representing the overlap between treatments in terms of identified proteins.

To identify differentially abundant proteins between treatments that are statistically significant, we performed a centered-log-ratio (CLR) transformation in R (version 4.0.2, compositions_2.0-1 package)[69] on peptide spectrum matches (PSMs) before performing statistical tests. We added 1 to every PSM value before performing the CLR transformation to protect against issues with missing values. Although CLR-normalized counts lose interpretability, CLR is a method better suited for statistical analyses of compositional data such as metaproteomics data [70,71]. Pairwise comparisons of all treatments were performed in the Perseus software platform (version 1.6.12.0) [42] using a Student's T-test corrected for multiple hypothesis testing with a permutation-based FDR of $5 \%(S 0=0.1$, both sides, not paired).

We used a principal component analysis (PCA) to visualize how samples separate or cluster based on relative protein abundances. We performed the analysis in the Perseus software platform (version 1.6.12.0) [42] on the CLR-transformed dataset described above.

We investigated whether the preservation treatment affected the measured abundances of specific taxa by comparing the relative biomass contributions of the taxa. Biomass contributions of specific taxa were 
assessed at the phylum and genus levels using the method described in Kleiner et al. [32]. Briefly, proteins were filtered for at least 2 protein unique peptides to increase the confidence in taxonomic identifications, and PSMs summed by taxon were used to estimate the biomass contribution of each taxon in the metaproteomes.

We investigated whether preservation treatments were biased towards proteins with specific biochemical characteristics such as isoelectric point ( $\mathrm{pl})$, molecular weight, or presence of transmembrane domains. We retrieved the $\mathrm{pl}$ and molecular weight associated with each identified protein from Proteome Discoverer and detected transmembrane domains by searching sequences of the identified proteins on the TMHMM 2.0 Server [72]. Then, we compared the distributions of these properties in each treatment as histograms with defined ranges.

Lastly, we assessed the amount of within-treatment variation using linear regression scatterplots in $\mathrm{R}$ (version 4. 0. 2; psych_2.1.3 package). We fit the scatterplots onto the percent normalized spectral abundance factors (\%NSAFs) for each pair of replicates that received the same treatment $(n=4$, except for the ethanol treatment time point 4 weeks: $n=3$ because sample E6 was removed). We then compared the Pearson correlation coefficients.

\section{Data availability}

The mass spectrometry metaproteomics data and protein sequence database were deposited to the ProteomeXchange Consortium via the PRIDE [73] partner repository with the dataset identifier PXD024115 [Reviewer Access at https://www.ebi.ac.uk/pride/login User: reviewer pxd024115@ebi.ac.uk Password: WVJJYH44]

\section{Results}

A fecal master mix (homogenate) was prepared from fecal samples of healthy adult conventional C57BL/6 mice. Aliquots of the master mix were randomized and then preserved using different methods. After 1 week and 4 weeks of storage, proteins were extracted and analyzed by LC-MS/MS. Preservation 
methods were assessed based on the amount of variability between replicates, and the degree of bias when compared to the other methods, particularly flash-freezing.

\section{Minimal differences in total numbers of identified features for co-extracted samples}

We compared the number of peptide spectrum matches (PSMs), peptides, proteins, and protein groups identified at a false discovery rate (FDR) of $5 \%$ between the different treatments and time-points (Figure 1) to determine whether the preservation treatment impacted the number of features identified. Samples that were preserved for 1 week and co-extracted as part of the first extraction batch did not significantly differ in their total counts, regardless of the preservation method. The numbers of features for the 4-week time-point (2nd extraction batch) and 1-week time-point differed slightly but differences did not test significant except for the samples preserved at $-80^{\circ} \mathrm{C}$ in RNAlater ${ }^{\mathrm{TM}}$ ("RF" samples). This difference is likely due to batch effects in sample preparation and peptide quantification via microBCA assay because the 1-week and 4-week samples were prepared separately. At the 4-week time-point, flash-frozen (FF) samples preserved for 4 weeks at $-80^{\circ} \mathrm{C}$ were significantly lower in their total counts compared to RF samples or samples preserved in the NAP buffer (N) or autoclaved NAP buffer (AN) at room temperature. 

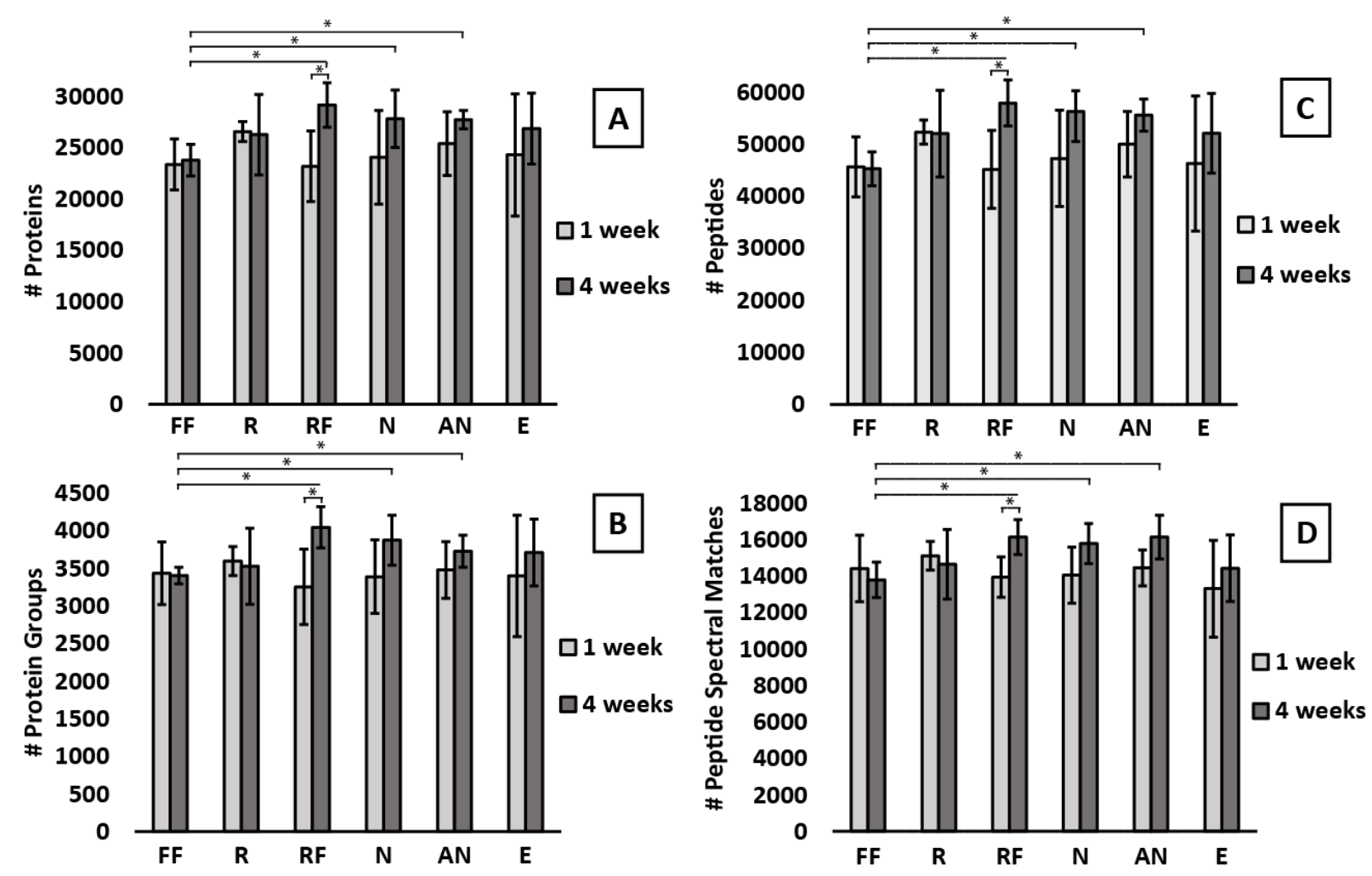

Figure 1. There were no significant differences in total numbers of PSMs, peptides, proteins, and protein groups between samples co-extracted after 1 week of preservation and only minimal differences existed in samples co-extracted after 4 weeks. $F F=$ Flash-freezing, $R=R N A l a t e r, R F=$ RNAlater + flash freezing, $\mathrm{N}=\mathrm{NAP}$ buffer, $\mathrm{AN}=$ Autoclaved NAP buffer, $\mathrm{E}=95 \%$ Ethanol. 1 week $=$ preserved for one week and first extraction batch. 4 weeks $=$ preserved for 4 weeks and second extraction batch. Bars represent the arithmetic mean $(n=4$ for all except $E-4$ weeks where $n=3)$. Error bars represent standard deviation. Asterisks indicate statistical significance (t-test, p-value $<0.05)$. A) Total proteins identified at 5\% FDR include the microbial, host, and dietary proteins. B) Total protein groups identified at 5\% FDR. C) Total peptides identified at 5\% FDR. D) Total peptide spectrum matches (PSMs) identified at 5\% FDR.

\section{Treatments shared over $76 \%$ of protein identifications}

Comparing and quantifying the proteins identified by multiple treatments showed that most proteins were detected in every treatment. Figure 2A shows a Venn diagram of the four most distinct treatments in terms of physical and chemical properties: R, E, FF, and N. Each of these 4 treatments produced metaproteomes that identified the same 4,641 proteins ( $76.3 \%$ of the dataset) and uniquely identified $\sim 1 \%$ of proteins. In the $E$ treatment, 281 proteins or $4.6 \%$ of the protein identifications were not detected; this was the largest proportion of undetected proteins, followed by the FF treatment that did not detect 198 proteins or $3.3 \%$ of the protein identifications. Figure 2B represents the overlap of proteins between 
chemically similar treatments: R, RF, N, and AN. Each of these 4 treatments produced metaproteomes that identified the same 4,878 proteins $(80.4 \%$ of the dataset) and uniquely identified $\sim 1 \%$ of proteins.

The RF treatment was the most distinct of the comparison shown in Figure $\mathbf{2 B}(\mathrm{R}, \mathrm{RF}, \mathrm{N}, \mathrm{AN})$ with 74 unique protein identifications $(\sim 1.2 \%)$ that were not detected in the other treatments.
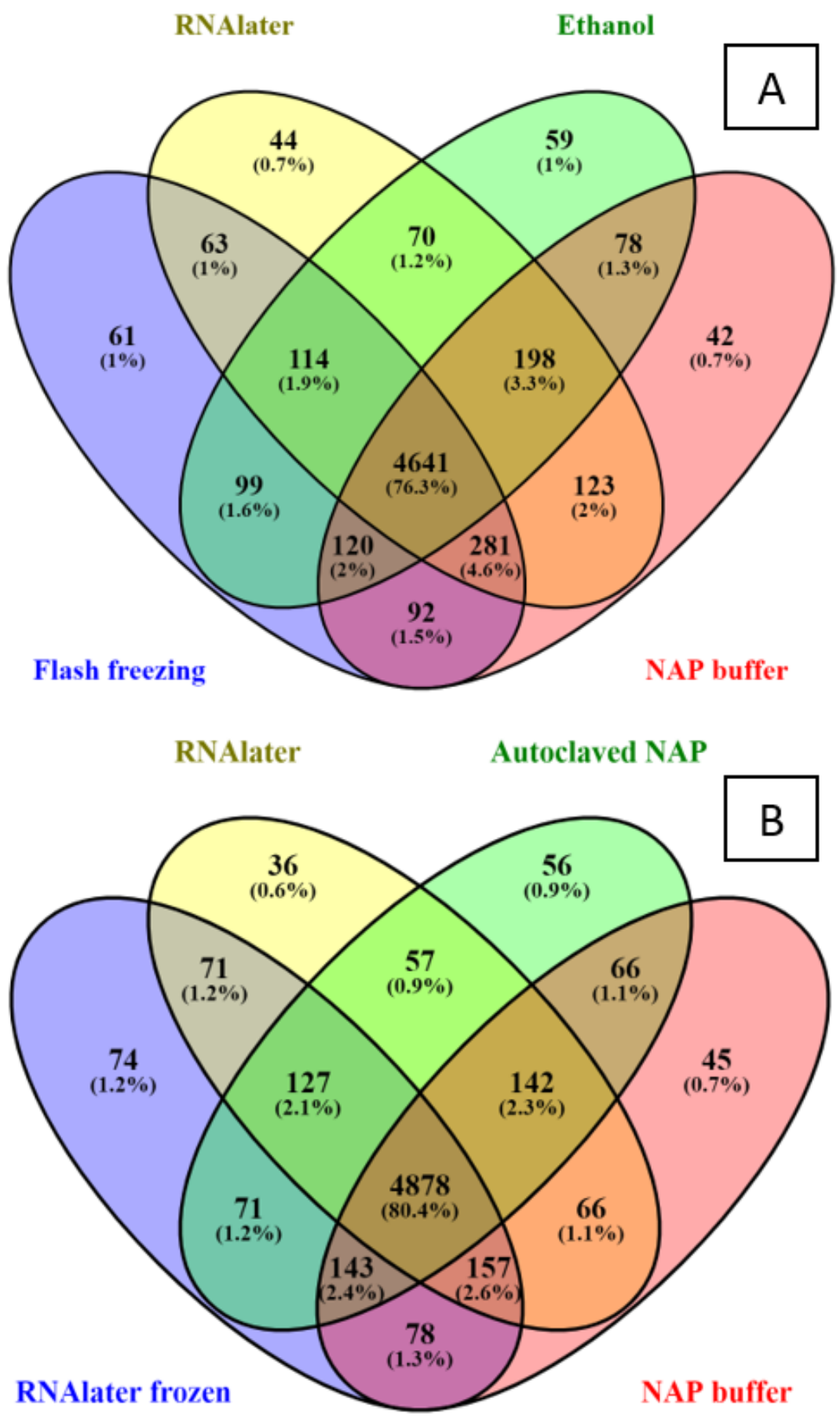
Figure 2. Over $76 \%$ of microbial, host and dietary protein identifications overlapped between treatments. Replicates of both time points were combined $(n=8$ samples per treatment, except for Ethanol where $n=7$ ). Proteins were included if they were identified with FDR $<5 \%$, at least 1 protein unique peptide, and present in at least 3 samples in the whole dataset. A) Comparison of treatments that differed most in terms of physical/chemical properties: Flash freezing (FF), RNAlater (R), 95\% Ethanol $(E)$, and NAP buffer (N). B) Comparison of the chemically similar treatments (RNAlater ${ }^{\mathrm{TM}}$ and RNAlater-like treatments): RNAlater (R), RNAlater frozen (RF), NAP buffer (N), Autoclaved NAP (AN).

\section{Relative protein abundances were highly similar between all treatments except for the ethanol treatment}

Principal component analysis (PCA), performed on the Centered-Log-ratio (CLR) transformed dataset of relative protein abundances, showed that the ethanol-preserved samples clustered together and clearly separated from samples of all other treatments (Figure 3A). If the preservation treatment did not affect protein profiles, we would expect to see no clustering, but rather the samples would be randomly distributed over the PCA plot.

We then compared CLR-transformed relative protein abundances between treatments using t-tests corrected for multiple hypothesis testing (two-sided, FDR of 0.05 and S0 of 0.1 ) to identify proteins that significantly differed in abundance based on the preservation treatment (Figure 3B). We found no significant differences between RNAlater ${ }^{\mathrm{TM}}(\mathrm{R})$ and the NAP buffer $(\mathrm{N})$ or between the NAP buffer $(\mathrm{N})$ and the autoclaved NAP buffer (AN). The proteins not shared between these treatments (Fig 2A) were sparse and lowly abundant proteins that were not significantly different from an undetected protein (Additional

file 2). The ethanol treatment was the most distinct treatment with, on average, about $9.5 \%$ of the proteins significantly differing in abundance, with 247 proteins being less abundant, as compared to the other treatments (Additional file 2). Flash-frozen samples and the samples preserved in RNAlater or RNAlater-like solutions produced metaproteomes that differed only minimally $(<1 \%$ of proteins with different abundances). 


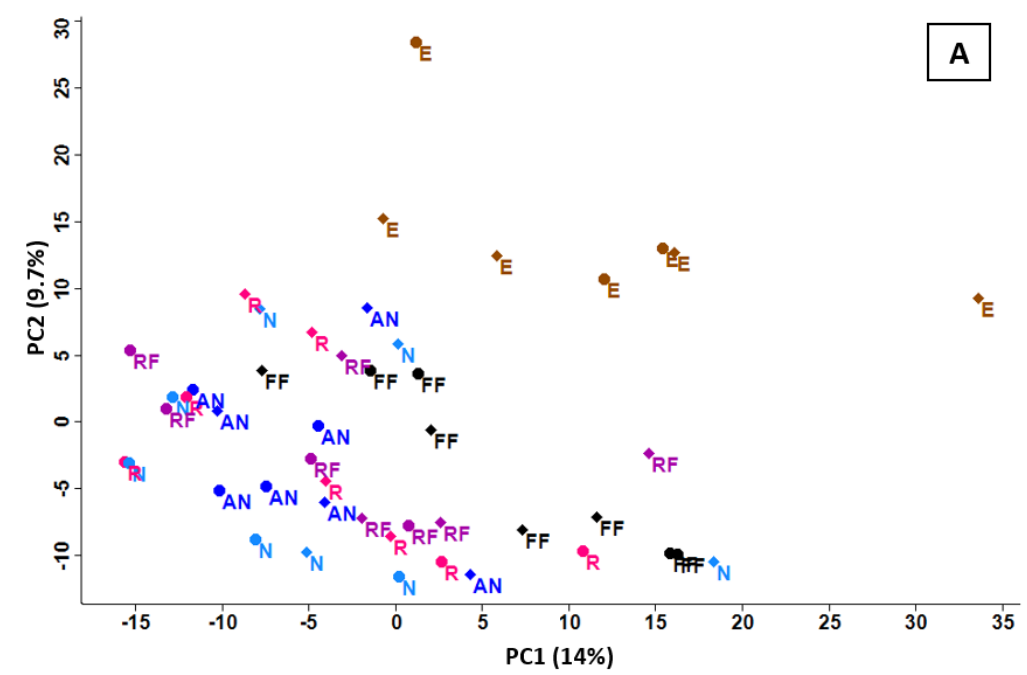

\begin{tabular}{l|lllll|} 
& RF & R & N & AN & E \\
\hline FF & $17(\sim 0.28 \%)$ & $41(\sim 0.67 \%)$ & $16(\sim 0.26 \%)$ & $30(\sim 0.49 \%)$ & $474(\sim 7.79 \%)$ \\
RF & & 0 & $2(\sim 0.03 \%)$ & 0 & $499(\sim 8.20 \%)$ \\
R & & & 0 & 0 & $556(\sim 9.14 \%)$ \\
N & & & & 0 & $683(\sim 11.22 \%)$ \\
AN & & & & & $680(\sim 11.17 \%)$
\end{tabular}

Figure 3. Ethanol preserved samples were distinct from all other samples in their protein abundance profiles. A) Principal component analysis (PCA) of the relative protein abundances from each sample (CLR-transformed). $\mathrm{E}=$ ethanol; $\mathrm{FF}=$ flash-freezing; $\mathrm{R}=\mathrm{RNA} / \mathrm{ate} \mathrm{r}^{\mathrm{TM}} ; \mathrm{RF}=\mathrm{RNA}$ /ater ${ }^{\mathrm{TM}}$ and flash-freezing; $\mathrm{N}=$ NAP buffer; AN = autoclaved NAP buffer. Diamonds = 1-week; Circles = 4-weeks. B) Number of significant differences between each treatment (two-sided t-test, FDR of 0.05 and S0 of 0.1). A significant difference represents one protein that is more abundant in one treatment over the other for each paired comparison (refer to Additional file 2 for directionality). Percentages in parentheses indicate the percentage of significant proteins out of the total proteins considered $(n=6,086)$.

\section{Within-treatment variability of relative protein abundances was low}

We assessed the amount of within-treatment variability in terms of quantified protein abundances by fitting linear scatter plots for all replicates against all replicates and evaluating the Pearson correlation coefficients (Additional file 6: Figures S1 - 6). The means of the Pearson correlation coefficients (Table 1) showed high correlation between replicates, indicating within treatment variability was low for all treatments.

\begin{tabular}{|l|l|l|}
\hline & 1 week & 4 weeks \\
\hline
\end{tabular}




\begin{tabular}{|l|l|l|}
\hline NAP buffer & $0.937+/-0.024$ & $0.963+/-0.006$ \\
\hline Autoclaved NAP buffer & $0.965+/-0.008$ & $0.958+/-0.02$ \\
\hline RNAlater ${ }^{\mathrm{TM}}$ & $0.957+/-0.01$ & $0.957+/-0.017$ \\
\hline RNAlater ${ }^{\mathrm{TM}}$ + Flash-freezing & $0.938+/-0.034$ & $0.967+/-0.008$ \\
\hline Flash Freezing & $0.961+/-0.009$ & $0.943+/-0.019$ \\
\hline 95\% Ethanol & $0.957+/-0.01$ & $0.95+/-0.015$ \\
\hline
\end{tabular}

Table 1. Linear correlation of replicates. Mean Pearson coefficient of the linear correlation between replicates of the same treatment and preservation duration $(n=4$, except for $E 4$ weeks: $n=3)$. We fit a linear model for each pair of samples within a treatment and time point in R (version 4. 0. 2; psych_2.1.3 package) using the dataset of percent normalized spectral abundance factors (\%NSAFs).

\section{Small but significant differences in the taxonomic composition of the metaproteomes based on the preservation method}

The relative taxonomic composition of the samples in terms of proteinaceous biomass contribution was consistent across replicates and preservation treatments (Additional text and additional. fig. S7). The biomass contribution is shown per phylum in Fig. 4A and per genus in Fig. 4B for the most abundant genera: Clostridium, Eubacterium, Butyrivibrio, Lactobacillus, Turicibacter, Blautia, Roseburia, and Coprococcus. The abundances of specific taxa significantly differed at the phylum and genus levels. At the phylum level, Firmicutes was overrepresented in the ethanol-preserved samples as compared to the flash-frozen and NAP-buffer preserved samples. At the genus level, Clostridium and Blautia were subtly but significantly overrepresented in the ethanol-preserved samples as compared to all other treatments (t-test, paired, 2-tailed, $\mathrm{p}<0.05)$. Furthermore, NAP buffer and Ethanol-preserved samples differed in their representation of the genus Butyrivibrio, and RNAlater and Ethanol-preserved samples differed in their representation of the genus Roseburia. 

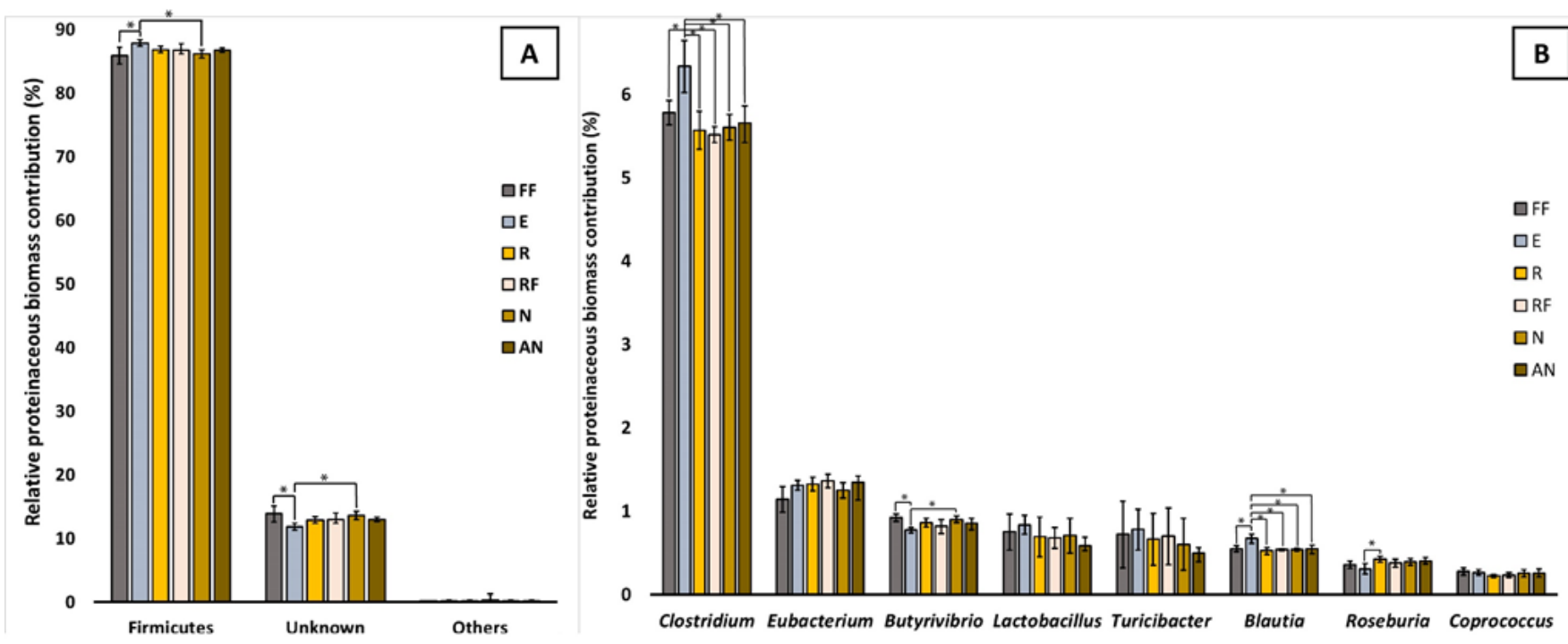

Figure 4. Small but significant differences in the representation of microbial taxa in the metaproteomes based on preservation method. Bars represent the mean percent proteinaceous biomass for each taxon at the phylum level (A) or the genus level (B). Error bars represent the standard deviation ( $n=8$, except $n=7$ for the Ethanol treatment). Asterisks represent statistical significance (t-test, paired, 2-tailed, $p<0.05)$. $E=$ ethanol; $F F=$ flash-freezing; $R=R N A l a t e r ; R F=R N A l a t e r$ and flash-freezing; $N=$ NAP buffer; AN = autoclaved NAP buffer. The eight most abundant genera are displayed in the figure. Percentages are low because genus-level taxonomy could be assigned for 11.1 $+/-0.53 \%(n=47)$ of the total proteinaceous biomass in our samples, distributed over 28 different microbial genera.

The preservation methods did not bias towards specific biochemical properties of proteins.

We investigated whether the preservation treatment biased towards or against proteins with a specific isoelectric point ( $\mathrm{pl}$ ), molecular weight (Additional Data 3), or transmembrane domains (Additional Data 4) by comparing the distributions of these properties in each treatment. Distributions did not differ between treatments, indicating no bias (Fig. 5). 

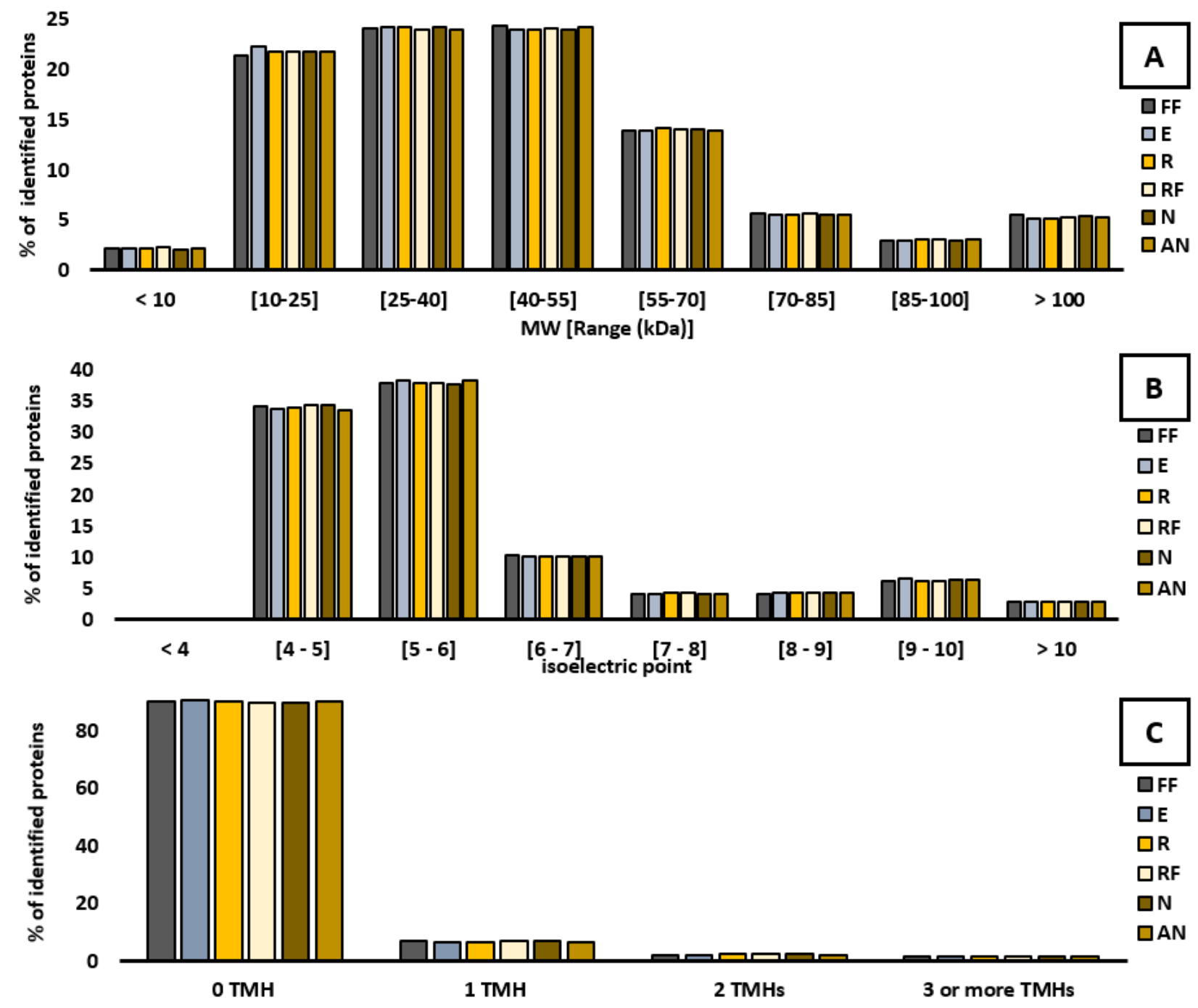

Figure 5. Distribution of biochemical properties of identified proteins. A) molecular weight (kDa) $B$ ) isoelectric point $C$ ) number of predicted transmembrane helices. Bars represent the proportion $(\%)$ of identified proteins belonging in each range. $E$ = ethanol; $F F=$ flash-freezing; $R=R N A l a t e r ; R F=$ RNAlater and flash-freezing; N = NAP buffer; AN = autoclaved NAP buffer.

\section{Discussion}

This study evaluated the effects of different preservation treatments on the metaproteomes of intestinal microbiome samples to identify a preservation method suitable to use when flash-freezing is not an option. The data show that the metaproteomes of samples preserved at room temperature while immersed in RNAlater ${ }^{\mathrm{TM}}$ or RNAlater-like solutions (NAP buffer and autoclaved NAP buffer) were highly similar to the metaproteomes of samples preserved by flash-freezing and storage at $-80^{\circ} \mathrm{C}$, with only 
negligible differences. On the other hand, samples preserved by immersion in $95 \%$ ethanol differed substantially from the flash-frozen samples and other samples. Because methods sharing the largest number of discoveries/values with most of the other methods tested may be more likely to produce valid results [74], our results suggest that the $95 \%$ ethanol treatment creates the largest bias in the metaproteomes. In contrast, the flash freezing, RNAlater ${ }^{\mathrm{TM}}$, and RNAlater-like treatments are most likely to represent the protein profiles at the time of collection accurately. The majority of the differences of the ethanol treatment were found at the protein abundance level. Roughly $9.5 \%$ of all the proteins in the ethanol preserved samples differed in abundance from all other samples, suggesting that ethanol preservation could strongly influence study results. Taxonomic abundances also differed under the ethanol treatment with the genera Clostridium and Blautia being overrepresented compared to all other treatment samples. However, although ethanol preservation introduces biases, within-treatment variability was low in ethanol-preserved samples. These results suggest that ethanol may be appropriate in some studies if it is used consistently.

While we tested a diversity of popular preservation methods, there are potentially other storage solutions and methods that could be used in addition to or instead of those tested and described herein. There are, for example, a range of commercially available "microbiome" storage solutions designed for preservation of fecal material for amplicon sequencing. These solutions could potentially also be used for metaproteomics, however, their compatibility with the proteomic workflow and quality of preservation would have to be carefully tested, particularly as compatibility issues could arise if a preservation reagent is incompatible with standard proteomic workflows. For example, some preservatives contain guanidinium chloride, which forms a solid if it contacts SDT lysis buffer [4\% (w/v) SDS, $100 \mathrm{mM}$ Tris-HCl pH 7.6, $0.1 \mathrm{M}$ DTT] that is used in many metaproteomics workflows.

Our results concur with the study by Saito et al. [55], which investigated sample preservation effects on the proteome of the cyanobacterium Synechococcus WH8102. They found RNAlater ${ }^{\mathrm{TM}}$ to be effective at preserving the proteome of a pure culture when compared to frozen storage. Here, we demonstrated that RNAlater ${ }^{\mathrm{TM}}$ can also be effective at preserving the metaproteomes of complex microbiome samples while minimizing storage effects. Furthermore, it appears that using the cost-effective RNAlater-like solution 
"NAP buffer" is a suitable alternative to the commercial RNAlater ${ }^{\mathrm{TM}}$ solution. Menke et al. [58] previously demonstrated that the NAP buffer effectively preserves the DNA of intestinal microbiome samples. Here we demonstrated that the NAP buffer effectively preserved proteins of intestinal microbiome samples and did not affect protein abundances when compared to samples preserved in RNAlater ${ }^{\mathrm{TM}}$. Furthermore, autoclaving the NAP buffer did not significantly affect the metaproteomes, suggesting that an autoclaved NAP buffer could be used in studies that require sterile material (e.g., gnotobiotic isolators).

\section{Conclusions}

This study evaluated the effects of different preservation treatments on the metaproteomes of intestinal microbiome samples. Based on our results, we recommend preserving intestinal microbiome samples by freezing, or in RNAlater ${ }^{\mathrm{TM}}$, or an RNAlater-like solution before metaproteomics analyses. The consistent use of these methods appears to minimize storage effects and thus improve the reliability of metaproteomics studies of the intestinal microbiome.

\section{Declarations}

\section{Ethics Approval}

The protocols for husbandry and experimentation of all mice used in this study were approved by the Institutional Animal Care and Use Committee at North Carolina State University (Institution reference: D16-00214).

\section{Availability of Data}

The mass spectrometry metaproteomics data and protein sequence database were deposited to the ProteomeXchange Consortium via the PRIDE [73] partner repository with the dataset identifier PXD024115 [Reviewer Access at https://www.ebi.ac.uk/pride/login User: reviewer pxd024115@ebi.ac.uk Password: WVJJYH44].

\section{Consent for publication}

Not applicable 


\section{Competing interests}

The authors declare no competing interests.

\section{Funding}

This work was supported by the National Institute Of General Medical Sciences of the National Institutes of Health under Award Number R35GM138362 and the Foundation for Food and Agriculture Research (FFAR) Grant ID: 593607

\section{Authors' contributions}

A.M. and M.K. designed the study. A.M. performed the experiments. A.M. and M.K. analyzed the data and wrote the manuscript.

\section{Acknowledgments}

We thank Deniz Durmusoglu for providing the fresh mouse fecal samples, Dr. Fernanda Salvato for providing A.M. with extensive metaproteomics training, Ibrahim Al'Abri and Alexandria Bartlett for providing mouse fecal samples for prior optimization rounds of the study, Marlene Jensen, Clara Tang, and Amelia Ellis who provided assistance in the lab, everyone in the Kleiner Lab for discussing the experimental design and results, and Dr. Heather Maughan for feedback on the manuscript. We made all LC-MS/MS measurements in the Molecular Education, Technology, and Research Innovation Center (METRIC) at NC State University.

\section{References}

1. Sommer F, Bäckhed F. The gut microbiota - masters of host development and physiology. Nat Rev Microbiol. 2013;11:227-38.

2. Rowland I, Gibson G, Heinken A, Scott K, Swann J, Thiele I, et al. Gut microbiota functions: metabolism of nutrients and other food components. Eur J Nutr. 2018;57:1-24.

3. Gentile CL, Weir TL. The gut microbiota at the intersection of diet and human health. Science. 2018;362:776-80.

4. Wikoff WR, Anfora AT, Liu J, Schultz PG, Lesley SA, Peters EC, et al. Metabolomics analysis reveals large effects of gut microflora on mammalian blood metabolites. Proc Natl Acad Sci. 2009;106:3698-703.

5. Turnbaugh PJ, Ley RE, Mahowald MA, Magrini V, Mardis ER, Gordon JI. An obesity-associated gut 
microbiome with increased capacity for energy harvest. Nature. 2006;444:1027-31.

6. Boursier J, Mueller O, Barret M, Machado M, Fizanne L, Araujo-Perez F, et al. The severity of nonalcoholic fatty liver disease is associated with gut dysbiosis and shift in the metabolic function of the gut microbiota. Hepatology. 2016;63:764-75.

7. Theriot CM, Koenigsknecht MJ, Carlson PE, Hatton GE, Nelson AM, Li B, et al. Antibiotic-induced shifts in the mouse gut microbiome and metabolome increase susceptibility to Clostridium difficile infection. Nat Commun. 2014;5:3114.

8. Montgomery TL, Künstner A, Kennedy JJ, Fang Q, Asarian L, Culp-Hill R, et al. Interactions between host genetics and gut microbiota determine susceptibility to CNS autoimmunity. Proc Natl Acad Sci. 2020;117:27516-27.

9. Belkaid Y, Hand TW. Role of the microbiota in immunity and inflammation. Cell. 2014;157:121-41.

10. Hooper LV, Littman DR, Macpherson AJ. Interactions between the microbiota and the immune system. Science. 2012;336:1268-73.

11. Chevalier G, Siopi E, Guenin-Macé L, Pascal M, Laval T, Rifflet A, et al. Effect of gut microbiota on depressive-like behaviors in mice is mediated by the endocannabinoid system. Nat Commun.

2020;11:1-15.

12. Rogers GB, Keating DJ, Young RL, Wong M-L, Licinio J, Wesselingh S. From gut dysbiosis to altered brain function and mental illness: mechanisms and pathways. Mol Psychiatry. 2016;21:738.

13. Ley RE, Bäckhed F, Turnbaugh P, Lozupone CA, Knight RD, Gordon JI. Obesity alters gut microbial ecology. Proc Natl Acad Sci. 2005;102:11070-5.

14. David LA, Maurice CF, Carmody RN, Gootenberg DB, Button JE, Wolfe BE, et al. Diet rapidly and reproducibly alters the human gut microbiome. Nature. 2014;505:559-63.

15. Turnbaugh PJ, Ridaura VK, Faith JJ, Rey FE, Knight R, Gordon JI. The effect of diet on the human gut microbiome: a metagenomic analysis in humanized gnotobiotic mice. Sci Transl Med. 2009;1:6ra14.

16. Bajaj JS, Heuman DM, Hylemon PB, Sanyal AJ, White MB, Monteith P, et al. Altered profile of human gut microbiome is associated with cirrhosis and its complications. J Hepatol. 2014;60:940-7.

17. Blakeley-Ruiz JA, Erickson AR, Cantarel BL, Xiong W, Adams R, Jansson JK, et al. Metaproteomics reveals persistent and phylum-redundant metabolic functional stability in adult human gut microbiomes of Crohn's remission patients despite temporal variations in microbial taxa, genomes, and proteomes. 
Microbiome. 2019;7:1-15.

18. Patnode ML, Beller ZW, Han ND, Cheng J, Peters SL, Terrapon N, et al. Interspecies competition impacts targeted manipulation of human gut bacteria by fiber-derived glycans. Cell. 2019;179:59-73.

19. Wang Y, Zhou Y, Xiao X, Zheng J, Zhou H. Metaproteomics: A strategy to study the taxonomy and functionality of the gut microbiota. J Proteomics. 2020;219:103737.

20. Heintz-Buschart A, Wilmes P. Human gut microbiome: function matters. Trends Microbiol. 2018;26:563-74.

21. Jansson JK, Baker ES. A multi-omic future for microbiome studies. Nat Microbiol. 2016;1:1-3.

22. Hettich RL, Pan C, Chourey K, Giannone RJ. Metaproteomics: Harnessing the power of high performance mass spectrometry to identify the suite of proteins that control metabolic activities in microbial communities. Anal Chem. 2013;85:4203-14.

23. Salvato F, Hettich RL, Kleiner M. Five key aspects of metaproteomics as a tool to understand functional interactions in host-associated microbiomes. PLOS Pathog. 2021;17:e1009245.

24. Kleiner M. Metaproteomics: Much more than measuring gene expression in microbial communities. mSystems. 2019;4:e00115-19.

25. Starr AE, Deeke SA, Li L, Zhang X, Daoud R, Ryan J, et al. Proteomic and metaproteomic approaches to understand host-microbe interactions. Anal Chem. 2017;90:86-109.

26. Xiong W, Abraham PE, Li Z, Pan C, Hettich RL. Microbial metaproteomics for characterizing the range of metabolic functions and activities of human gut microbiota. Proteomics. 2015;15:3424-38.

27. Verberkmoes NC, Russell AL, Shah M, Godzik A, Rosenquist M, Halfvarson J, et al. Shotgun metaproteomics of the human distal gut microbiota. ISME J. 2009;3:179-89.

28. Zhang X, Chen W, Ning Z, Mayne J, Mack D, Stintzi A, et al. Deep metaproteomics approach for the study of human microbiomes. Anal Chem. 2017;89:9407-15.

29. Lee PY, Chin S-F, Neoh H, Jamal R. Metaproteomic analysis of human gut microbiota: where are we heading? J Biomed Sci. 2017;24:1-8.

30. Long S, Yang Y, Shen C, Wang Y, Deng A, Qin Q, et al. Metaproteomics characterizes human gut microbiome function in colorectal cancer. Npj Biofilms Microbiomes. 2020;6:1-10.

31. Zhang X, Deeke SA, Ning Z, Starr AE, Butcher J, Li J, et al. Metaproteomics reveals associations 
between microbiome and intestinal extracellular vesicle proteins in pediatric inflammatory bowel disease. Nat Commun. 2018;9:1-14.

32. Kleiner M, Thorson E, Sharp CE, Dong X, Liu D, Li C, et al. Assessing species biomass contributions in microbial communities via metaproteomics. Nat Commun. 2017;8:1558.

33. Pible O, Allain F, Jouffret V, Culotta K, Miotello G, Armengaud J. Estimating relative biomasses of organisms in microbiota using "phylopeptidomics." Microbiome. 2020;8:30.

34. Gouveia D, Pible O, Culotta K, Jouffret V, Geffard O, Chaumot A, et al. Combining proteogenomics and metaproteomics for deep taxonomic and functional characterization of microbiomes from a non-sequenced host. Npj Biofilms Microbiomes. 2020;6:1-6.

35. Van Den Bossche T, Kunath BJ, Schallert K, Schäpe SS, Abraham PE, Armengaud J, et al. Critical assessment of metaproteome investigation (CAMPI): a multi-lab comparison of established workflows. bioRxiv. 2021;:2021.03.05.433915.

36. Kleiner M, Kouris A, Jensen M, Liu Y, McCalder J, Strous M. Ultra-sensitive Protein-SIP to quantify activity and substrate uptake in microbiomes with stable isotopes. bioRxiv. 2021;:2021.03.29.437612.

37. Kleiner M, Dong X, Hinzke T, Wippler J, Thorson E, Mayer B, et al. Metaproteomics method to determine carbon sources and assimilation pathways of species in microbial communities. Proc Natl Acad Sci. 2018;115:E5576-84.

38. Justice N, Li Z, Wang Y, Spaudling SE, Mosier AC, Hettich RL, et al. (15)N- and (2)H proteomic stable isotope probing links nitrogen flow to archaeal heterotrophic activity. Environ Microbiol. 2014;16:3224-37.

39. Jehmlich N, Schmidt F, Hartwich M, Bergen M von, Richnow H-H, Vogt C. Incorporation of carbon and nitrogen atoms into proteins measured by protein-based stable isotope probing (Protein-SIP). Rapid Commun Mass Spectrom. 2008;22:2889-97.

40. Zhang X, Li L, Mayne J, Ning Z, Stintzi A, Figeys D. Assessing the impact of protein extraction methods for human gut metaproteomics. J Proteomics. 2018;180:120-7.

41. Tanca A, Palomba A, Fraumene C, Pagnozzi D, Manghina V, Deligios M, et al. The impact of sequence database choice on metaproteomic results in gut microbiota studies. Microbiome. 2016;4:1-13.

42. Tyanova S, Temu T, Sinitcyn P, Carlson A, Hein MY, Geiger T, et al. The Perseus computational platform for comprehensive analysis of (prote)omics data. Nat Methods. 2016;13:731-40.

43. Jordan S, Baker B, Dunn A, Edwards S, Ferranti E, Mutic AD, et al. Maternal-child microbiome: 
specimen collection, storage and implications for research and practice. Nurs Res. 2017;66:175.

44. Kim D, Hofstaedter CE, Zhao C, Mattei L, Tanes C, Clarke E, et al. Optimizing methods and dodging pitfalls in microbiome research. Microbiome. 2017;5:1-14.

45. Carruthers LV, Moses A, Adriko M, Faust CL, Tukahebwa EM, Hall LJ, et al. The impact of storage conditions on human stool 16S rRNA microbiome composition and diversity. PeerJ. 2019;7:e8133.

46. Hale VL, Tan CL, Knight R, Amato KR. Effect of preservation method on spider monkey (Ateles geoffroyi) fecal microbiota over 8 weeks. J Microbiol Methods. 2015;113:16-26.

47. Williams SCP. Gnotobiotics. Proc Natl Acad Sci. 2014;111:1661.

48. Fouhy F, Deane J, Rea MC, O'Sullivan Ó, Ross RP, O'Callaghan G, et al. The effects of freezing on faecal microbiota as determined using MiSeq sequencing and culture-based investigations. PLOS ONE. 2015;10:e0119355.

49. Carroll IM, Ringel-Kulka T, Siddle JP, Klaenhammer TR, Ringel Y. Characterization of the fecal microbiota using high-throughput sequencing reveals a stable microbial community during storage. PLOS ONE. 2012;7:e46953.

50. Tap J, Cools-Portier S, Pavan S, Druesne A, Öhman L, Törnblom H, et al. Effects of the long-term storage of human fecal microbiota samples collected in RNAlater. Sci Rep. 2019;9:1-9.

51. Blekhman R, Tang K, Archie EA, Barreiro LB, Johnson ZP, Wilson ME, et al. Common methods for fecal sample storage in field studies yield consistent signatures of individual identity in microbiome sequencing data. Sci Rep. 2016;6:1-5.

52. Song SJ, Amir A, Metcalf JL, Amato KR, Xu ZZ, Humphrey G, et al. Preservation methods differ in fecal microbiome stability, affecting suitability for field studies. mSystems. 2016;1:e00021-16.

53. Choo JM, Leong LE, Rogers GB. Sample storage conditions significantly influence faecal microbiome profiles. Sci Rep. 2015;5:1-10.

54. Passow CN, Kono TJY, Stahl BA, Jaggard JB, Keene AC, McGaugh SE. RNAlater and flash freezing storage methods nonrandomly influence observed gene expression in RNAseq experiments. bioRxiv. $2018 ;: 379834$.

55. Saito MA, Bulygin VV, Moran DM, Taylor C, Scholin C. Examination of microbial proteome preservation techniques applicable to autonomous environmental sample collection. Front Microbiol. 2011;2:215-215. 
56. Hickl O, Heintz-Buschart A, Trautwein-Schult A, Hercog R, Bork P, Wilmes P, et al. Sample preservation and storage significantly impact taxonomic and functional profiles in metaproteomics studies of the human gut microbiome. Microorganisms. 2019;7:367.

57. de Boer R, Peters R, Gierveld S, Schuurman T, Kooistra-Smid M, Savelkoul P. Improved detection of microbial DNA after bead-beating before DNA isolation. J Microbiol Methods. 2010;80:209-11.

58. Menke S, Gillingham MAF, Wilhelm K, Sommer S. Home-Made cost effective preservation buffer is a better alternative to commercial preservation methods for microbiome research. Front Microbiol.

2017;8:102.

59. Camacho-Sanchez M, Burraco P, Gomez-Mestre I, Leonard JA. Preservation of RNA and DNA from mammal samples under field conditions. Mol Ecol Resour. 2013;13:663-73.

60. Sinha R, Chen J, Amir A, Vogtmann E, Shi J, Inman KS, et al. Collecting fecal samples for microbiome analyses in epidemiology studies. Cancer Epidemiol Biomark Prev. 2016;25:407-16.

61. Wiśniewski JR, Zougman A, Nagaraj N, Mann M. Universal sample preparation method for proteome analysis. Nat Methods. 2009;6:359.

62. Speare L, Smith S, Salvato F, Kleiner M, Septer AN. Environmental viscosity modulates interbacterial killing during habitat transition. mBio. 2020;11:e03060-19.

63. Oberg AL, Vitek O. Statistical design of quantitative mass spectrometry-based proteomic experiments. J Proteome Res. 2009;8:2144-56.

64. Xiao L, Feng Q, Liang S, Sonne SB, Xia Z, Qiu X, et al. A catalog of the mouse gut metagenome. Nat Biotechnol. 2015;33:1103-8.

65. Timmins-Schiffman E, May DH, Mikan M, Riffle M, Frazar C, Harvey HR, et al. Critical decisions in metaproteomics: achieving high confidence protein annotations in a sea of unknowns. ISME J.

2017;11:309-14.

66. Li W, Godzik A. Cd-hit: a fast program for clustering and comparing large sets of protein or nucleotide sequences. Bioinforma Oxf Engl. 2006;22:1658-9.

67. Zybailov B, Mosley AL, Sardiu ME, Coleman MK, Florens L, Washburn MP. Statistical analysis of membrane proteome expression changes in saccharomyces cerevisiae. J Proteome Res. 2006;5:2339-47.

68. Oliveros, J.C. Venny. An interactive tool for comparing lists with Venn's diagrams. 2015. 
https://bioinfogp.cnb.csic.es/tools/venny/index.html.

69. Aitchison J. The statistical analysis of compositional data. J R Stat Soc. 1982;44:139-77.

70. Fernandes AD, Reid JN, Macklaim JM, McMurrough TA, Edgell DR, Gloor GB. Unifying the analysis of high-throughput sequencing datasets: characterizing RNA-seq, 16S rRNA gene sequencing and selective growth experiments by compositional data analysis. Microbiome. 2014;2:1-13.

71. Gloor GB, Macklaim JM, Pawlowsky-Glahn V, Egozcue JJ. Microbiome datasets are compositional: and this is not optional. Front Microbiol. 2017;8:2224.

72. Krogh A, Larsson B, von Heijne G, Sonnhammer ELL. Predicting transmembrane protein topology with a hidden Markov model: application to complete genomes. J Mol Biol. 2001;305:567-80.

73. Vizcaíno JA, Csordas A, del-Toro N, Dianes JA, Griss J, Lavidas I, et al. 2016 update of the PRIDE database and its related tools. Nucleic Acids Res. 2016;44:D447-56.

74. Calgaro M, Romualdi C, Waldron L, Risso D, Vitulo N. Assessment of single cell RNA-seq statistical methods on microbiome data. bioRxiv. 2020;:2020.01.15.907964. 